\title{
DETERMINATION OF ORGAN VOLUME USING FOCUSED IMPEDANCE METHOD (FIM): A SIMULATION APPROACH
}

\author{
Sayed Parvez Ahmed ${ }^{1,2}$, M. Abdul Kadir ${ }^{1 *}$, Rubina Rahman ${ }^{2}$, Golam Dastegir Al-Quaderi ${ }^{3}$ and \\ K. Siddique-e Rabbani ${ }^{1}$ \\ ${ }^{1}$ Department of Biomedical Physics \& Technology, University of Dhaka, Dhaka 1000, Bangladesh \\ ${ }^{2}$ Department of Physics, Jahangirnagar University, Savar, Dhaka, Bangladesh \\ ${ }^{3}$ Department of Physics, University of Dhaka, Dhaka 1000, Bangladesh \\ * Corresponding author email: kadir@du.ac.bd,
}

\begin{abstract}
A noninvasive and radiation free technique for in-vivo measurement of the volume of organs or fluids in the human body is necessary for many clinical applications. Focused Impedance Method (FIM) is a novel technique of electrical impedance measurements which has enhanced sensitivity in a localized region. FIM can sense the change in transfer impedance of an organ within a reasonable depth of the human body using surface electrodes, minimizing contributions from its neighbouring regions. This of course assumes that the impedance properties of the embedded object are different from that of its surrounding tissues. This paper presents a new method for the determination of the volume of an organ within body using dual electrode separations of a concentric 4-electrode FIM configuration. In order to develop this formalism simulated FIM measurements using surface electrodes on a cubic volume conductor with embedded spherical objects were performed using a Finite Element (FE) based simulation software, COMSOL Multiphysics ${ }^{\circledR}$. For the present methodology, the conductivity of the object with respect to its surroundings and its depth need to be known. The former is obtainable through some primary invasive or in vivo measurements while the latter may be approximated using anatomy. Experimental results on a phantom made up of a cubic tank filled with saline showed that the proposed method can be used to determine the volume of embedded objects to an accuracy of about $5 \%$ which is adequate for most physiological measurements. The technique may also find use in geology, oceanography and industry.
\end{abstract}

Keywords: Organ Volume, Volume Determination, Focused Impedance Method, FIM, Electrical Impedance

\section{INTRODUCTION}

A measure of the volume of organs, masses or fluids within the body is of great importance both for evaluation and management of physiological disorders in the human body. For example, determination of the thyroid gland volume is essential for proper diagnosis and therapy [Tsuruta et al 1993, Hansen et al 1979]. Similarly, the volume of acid secreted in the stomach [Hersey and Sachs 1995] needs to be measured for evaluation of gastric functionality [Ryle 1926, Howden and Hunt 1987]. Volumes of kidney, liver and prostate are indicative of several life threatening diseases [Grantham et al 2006, Shoup et al 2003, Chen et al 1999]. Progression of tumour within body can also be monitored by determining its volume. Computed tomography and isotopic methods are in use for estimation of in vivo organ volume [Geraghty et al 2004, Tauxe et al 1982, Heymsfield et al 1979]. However, these methods involve radiation hazards. Measurement of organ volume using ultrasonography is known to be safe although the presence of intra-thoracic components may lead to inaccurate results. Electrical impedance techniques have drawn attention of scientists over many years for physiological study and diagnosis as the human body is a volume conductor of electricity [Rigaud et al 1995]. Since organs, fluids or tumours within body possess different conductivity compared to its surrounding tissues; electrical methods have the potential to determine these volumes and the present 
work was taken up with this aim. Besides, non-invasive measurements were targeted, using skin surface electrodes.

Conventional tetra-polar (or four electrode) electrical impedance measurement (TPIM) is generally used for measurements on the human body [Lukaski and Bolonchuk 1988] as this can eliminate the contact potentials of the electrode-tissue interface which are particularly high for skin surface electrodes at low frequencies. Besides, the measurements are all performed using ac rather than dc to avoid electrochemical potentials and possible tissue injury. In the TPIM arrangement, an alternating current of constant amplitude is injected into a volume conductor through a pair of surface electrodes and the resulting voltage across another surface electrode pair is measured. The ratio of the measured voltage to the injected current gives the transfer impedance of the volume conductor for a particular electrode configuration [Brown et al 2000]. The extent to which a change in conductivity of a point within the volume conductor contributes to the measured transfer impedance is defined as the sensitivity of that point. One of the major disadvantages of TPIM technique is its extended spatial sensitivity distribution [Islam et al 2010] and therefore is not suitable for localized measurements. Electrical Impedance Tomography (EIT) has shown the promise of conductivity imaging which may be used to have localized information [Holder 2010]. However, the resolution that EIT can offer is poor and dependent on number of electrodes. Moreover, the effect of $3^{\text {rd }}$ dimension may give erroneous image [Rabbani and Kabir 1991]. EIT requires many electrodes making it complex from a practical point of view.

Focused Impedance Method (FIM), an innovation of our extended group at the University of Dhaka, uses only a few electrodes to obtain a localized sensitivity distribution. It can measure the change in transfer impedance of a specified target zone within a volume conductor minimizing the contribution from its neighbors [(Rabbani et al 1999, Rabbani and Karal 2008, Islam et al 2010, Kadir et al 2013, Kadir et al 2015]. It has three versions using 8, 6 and 4 electrodes. The basis of the FIM technique is that the impedance of the region of interest is measured along two mutually perpendicular directions giving an enhanced sensitivity in the central region compared to its surroundings. In 4-electrode FIM, electrodes are placed at the corners of a square region on the surface of a volume conductor. In the first step, an alternating current is driven through two adjacent electrode pair (drive electrodes) while the potential is measured across the other pair (receive electrodes). The ratio of measured voltage to the injected current gives the transfer impedance in one direction. Then a similar measurement is performed in an orthogonal direction and the sum of these two transfer impedance values gives the focused impedance (FZ). The FIM technique has already been applied successfully in the study of gastric emptying [Rabbani et al 1999], lung ventilation [Kadir et al 2010], abdominal fat thickness measurement [Surovy et al 2012], breast tumour characterization [Al Amin et al., 2014] and has potential application in other in other areas [Rabbani and Kadir 2011]. A preliminary simulation study has shown the potential of FIM in estimating the volume of insulating objects embedded within a volume conductor [Kadir et al 2013]. In the present study, the efficacy of using 4-electrode FIM for volume determination was investigated in detail considering both conducting and resistive objects located inside volume conductors. The study was performed using finite element based simulations which was then validated using phantom measurements.

\section{METHODS, OBSERVATION AND RESULTS}

The AC/DC module of the finite element based software Comsol Multiphysics ${ }^{\circledR}$ [Multiphysics 2012] was used to simulate the impedance measurements as described below. A cubic insulating tank with $0.3 \mathrm{~m}$ sides and filled with saline of conductivity $0.2 \mathrm{~S} / \mathrm{m}$ was modeled as the volume conductor. Four 
cylindrical electrodes of diameter $1 \mathrm{~cm}$ were placed at the corners of a square region, centrally on the top surface (figure 1) to configure for 4-electrode FIM measurement. The electrode arrangement is shown more clearly in figure 1 (right), marked A to D, the separation between the centers of the adjacent electrodes being termed as the Electrode Separation (ES). A spherical object of conductivity different from that of the background volume was placed within the volume conductor keeping its center below the center of the electrode configuration. The distance between the center of the object and the electrode plane was denoted as depth $(d)$. For simulated FIM measurements, an alternating
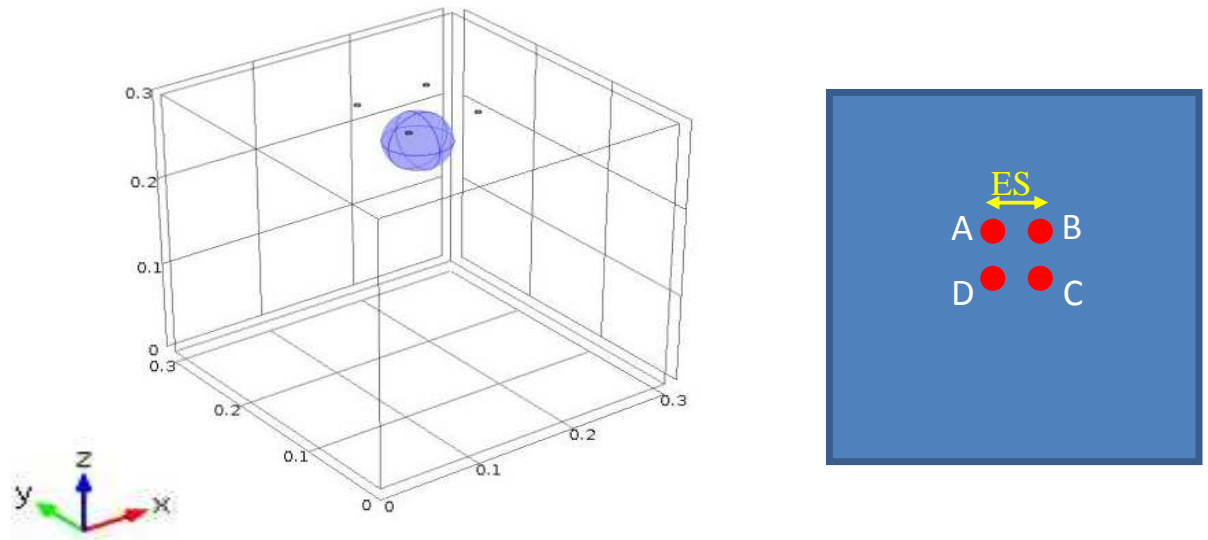

current of amplitude 1A was driven through the electrode pair $(\mathrm{A}, \mathrm{B})$ using the electric current (ec) interface of COMSOL AC/DC module. The receive electrodes (C,D) were then considered as boundary probes to measure the voltage difference developed. In the next sequence, the electrode pair $(\mathrm{A}, \mathrm{D})$ was used for current injection and the voltage was measured across the pair $(\mathrm{B}, \mathrm{C})$. Focused impedance $(F Z)$ was then calculated summing the two voltage values (although strictly it should be the average of the two values, for simplicity in measurement, the sum is considered). The simulation was performed in the frequency domain with a drive current frequency of $5 \mathrm{kHz}$. The governing equations for the FEM computations are,

$$
\begin{aligned}
& \nabla . J=Q_{j} \\
& J=\left(\sigma+j \omega \varepsilon_{0} \varepsilon_{r}\right) E+J_{e} \\
& E=-\nabla V
\end{aligned}
$$

where, $J, Q_{j}, \sigma, \omega, \varepsilon_{0}, \varepsilon_{r}, E, J_{e}$ and $V$ are the current density, source charge, electrical conductivity, angular frequency, permittivity in free space and relative permittivity of the material, electric field, external current density and electric potential respectively.

To investigate the effect of electrode separation $E S$, initially, the $F Z$ values of the volume conductor without any object was computed at different electrode separations. Then a spherical object of a certain diameter of conductivity $2.0 \mathrm{~S} / \mathrm{m}$ (10 times more conducting than the volume conductor) was placed at a depth $(d)$ from the electrode plane and $F Z$ values were computed with the same values of $E S$ as above keeping the object conductivity and depth constant. The difference between the corresponding $F Z$ values with and without object was termed $\triangle F Z$. Similar measurements for $\Delta F Z$ 
were performed for an object with conductivity $0.02 \mathrm{~S} / \mathrm{m}$ (10 times less conducting than the volume conductor). To understand the boundary effect, the above measurements were repeated for a larger cubic volume conductor of side $100 \mathrm{~cm}$ keeping all other parameters same. Similar measurements were then performed varying the conductivity of the target object with different radii $(r)$ and placed at different depths.

To validate the simulation results experimentally, a similar study was performed on an insulating cubic tank filled with saline. The dimensions of the tank were identical to the first sized tank $(0.3 \mathrm{~m}$ cube) used for simulation study. Spherical insulating balls were used as objects which were hung using fine insulating threads. The focused impedance values were measured at two electrode separations using a commercial four electrode impedance meter (Maltron Bioscan-II, UK).

The variations of $\triangle F Z$ against $E S$ for objects with conductivity 10 times and 0.1 times that of background and with different radii embedded in a cubic volume conductor of side $0.3 \mathrm{~m}$ are plotted in figure 2. $\triangle F Z$ is positive for less conducting objects and negative for more conducting objects. It can be observed that for all object sizes $\triangle F Z$ decreases with increasing $E S$. The decrease in $\triangle F Z$ is sharper at lower $E S$ and less sharp at higher electrode separations reaching an almost constant value. Intuitively, $\triangle F Z$ should approach zero at higher electrode separations but it is not so as can be seen in figure 2, even for $\mathrm{r}=0.03 \mathrm{~m}$ at $\mathrm{ES}=0.2 \mathrm{~m}$. This may be due to boundary effect of the volume conductor which is $0.3 \mathrm{~m}$ in this case. To investigate this phenomenon, the study was repeated on a cubic volume conductor of side $1.0 \mathrm{~m}$. Figure 3 shows the variation of $\triangle F Z$ against $E S$ for this larger volume conductor where the $\Delta F Z$ value is much less, almost zero at $E S=0.2 \mathrm{~m}$. Therefore, the nonzero values of $\triangle F Z$ at large $\mathrm{ES}$ in figure 2 were indeed due to boundary effect.

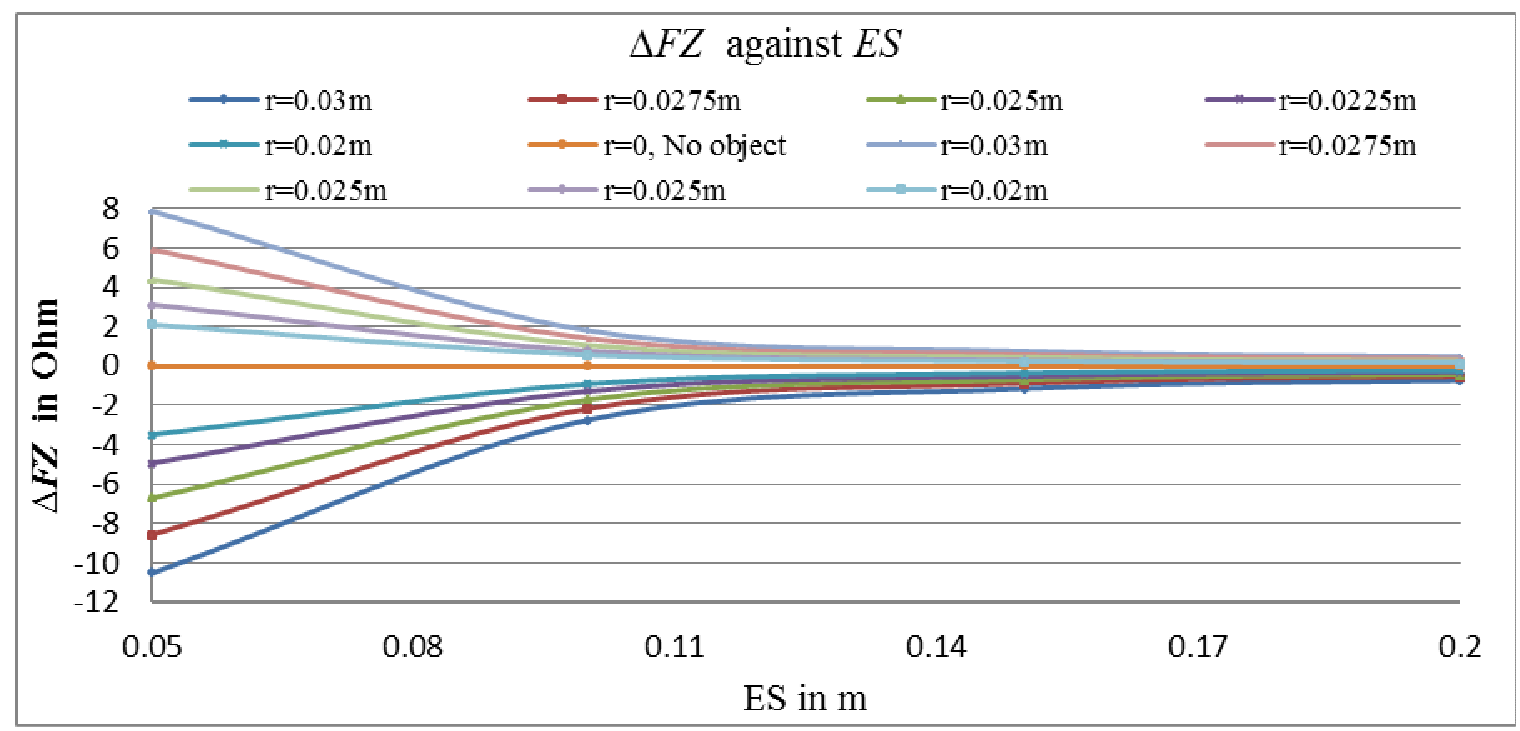

Figure 2: Variation of $\triangle F Z$ against $E S$ for objects of various radii embedded in a cubic volume conductor of edge length $0.3 \mathrm{~m}$. The curves above the horizontal line at $\triangle F Z=0$ are for objects with conductivity 10 times lower than that of the volume conductor. The curves below are for objects with conductivity 10 times greater (indicated $r$ values at the top, sequentially left to right, before $r=0$, are for the lower curves while those following $r=0$ are for the upper curves) 


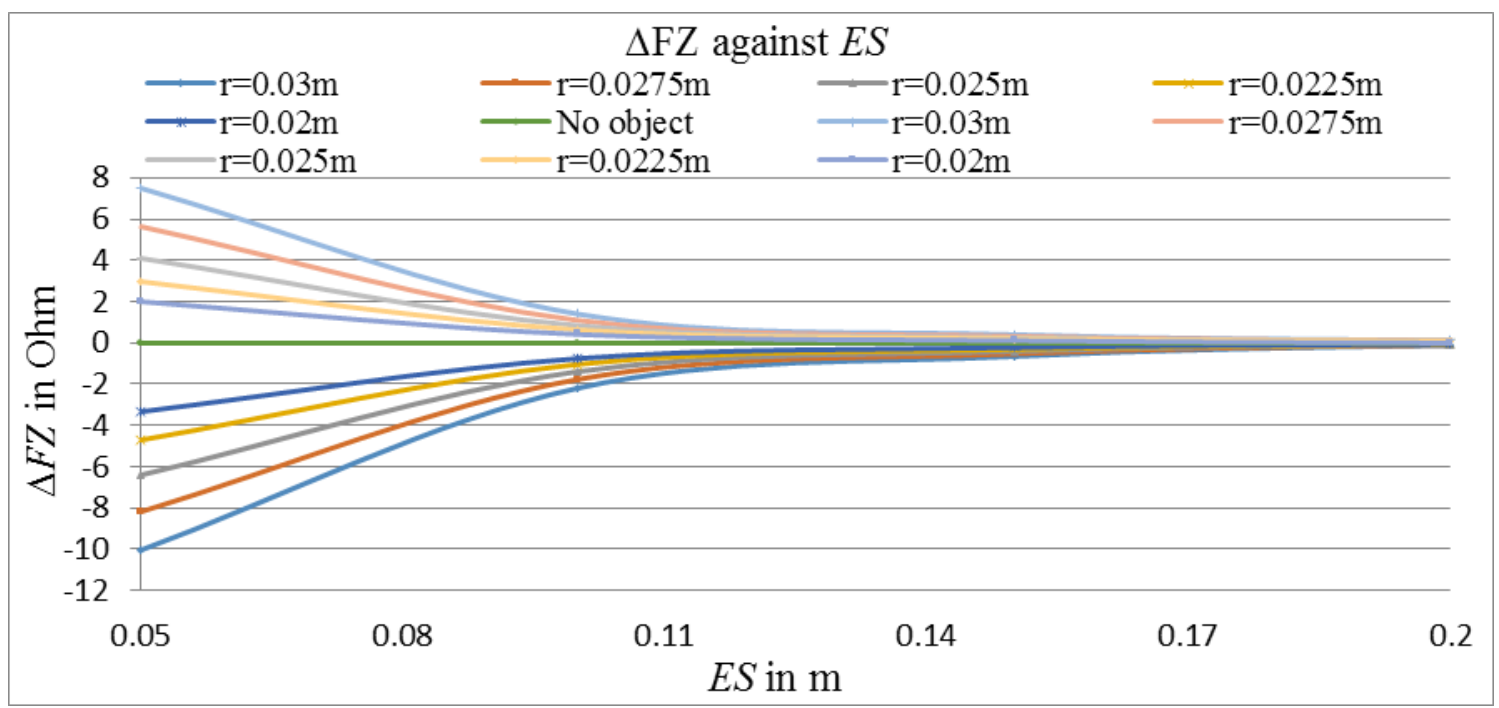

Figure 3 Similar graphs as in Fig 2, but with the volume conductor of larger size, with side $1.0 \mathrm{~m}$. The $\triangle F Z$ values at higher $E S$ are much lower, almost zero, as expected intuitively.

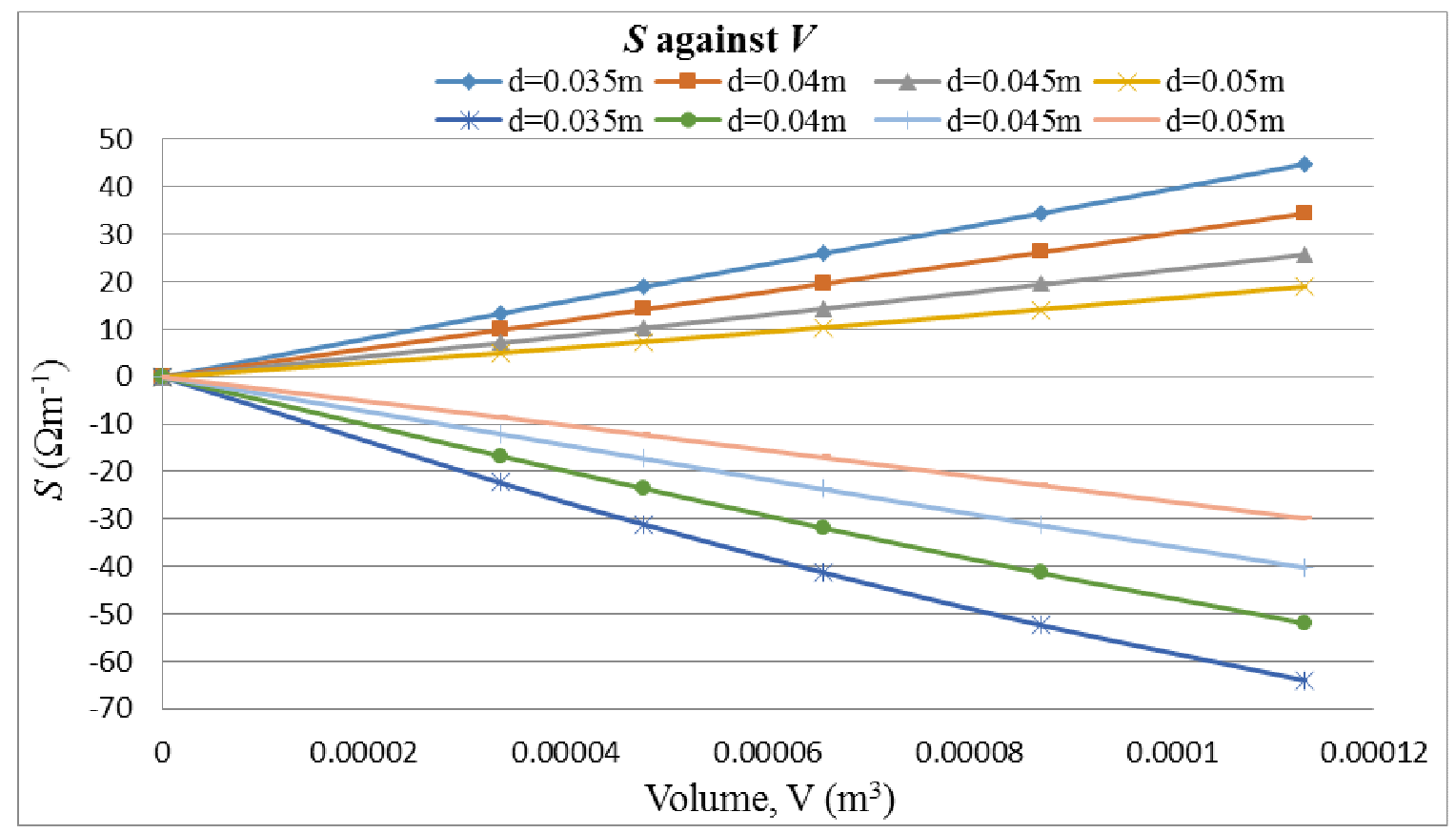

Figure 4: Slope (S) plotted against volume (V) of the embedded object at different depth (d). The curves above the horizontal line at $S=0$ are for objects with conductivity lower than that of the volume conductor. The curves below the horizontal line are for more conducting objects.

Figures 2 and 3 show that at lower electrode separations, the variations of $\triangle F Z$ with $E S$ are almost linear and the slopes of this linear part of the curves are higher for objects having greater radius. This indicates that the slope values may be used to determine the object volumes. So we defined a parameter, slope $(S)$ of the linear portion of the curves between two chosen electrode separations $E S_{2}$ and $E S_{l}$ as:

$$
S=\frac{\Delta F Z\left(E S_{1}\right)-\triangle F Z\left(E S_{2}\right)}{E S_{2}-E S_{1}}
$$


We then plotted the slope $S$ with the object volume, $V$ for different depths and these are shown in Figure 4. It is striking to observe that for a constant depth $(d), S$ varies linearly with volume of the object, and the slope of these lines in turn changes with the depth. The slope $S$ can be expressed mathematically as,

$S=A V$

where $A$ is the slope of a straight line corresponding to a particular depth. The value of $A$ is positive for objects with conductivity lower than that of the background of the volume conductor. On the other hand, the value of $A$ is negative for objects with conductivity higher than that of the volume conductor. The slope $A$ clearly on the depth of the object. It is also expected to depend on the conductivity and permittivity of the object and that of the volume conductor. From figure 4 it can be seen that the magnitude of $A$ decreases with increased depth of the object. To explore the relationship between $A$ and depth of the embedded object $d$, the magnitude of $A$ was plotted against inverse of depth in figure 5. The straight line fit indicates that $A$ is inversely proportional to $d$ which can be expressed mathematically as

$|A|=\frac{K}{d}+C$

where $\mathrm{K}$ and $\mathrm{C}$ are constants which depend on the conductivity and permittivity of the object and the volume conductor under investigation, dimension of the volume conductor and the electrodes used and the electrode separations chosen.

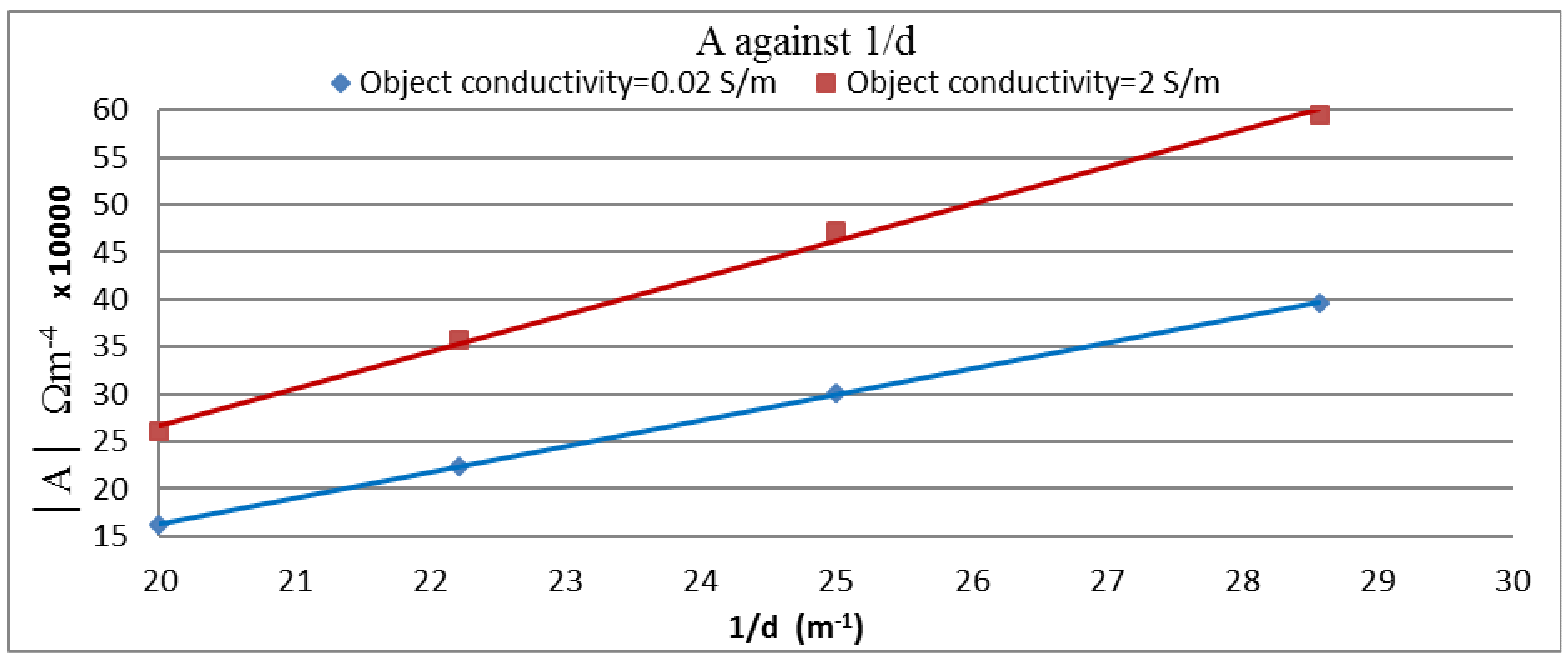

Figure 5: Variation of $|A|$ against inverse of depth both for conducting and resistive objects.

Equation (6) and (7) can be combined as

$$
V=\frac{s}{\left|\frac{K}{d}+c\right|}
$$

Which gives the volume of the embedded object in terms of other measured parameters. Therefore, the volume of an arbitrary object embedded in a volume conductor can be determined measuring the focused impedances at two electrode separations if the depth of the object and its impedivity are known. 


\section{PERFORMANCE EVALUATION}

To study the accuracy of the proposed method of volume determination using a COMSOL simulation, objects of different conductivity and volume were introduced within the volume conductor at a few

Table-1: Comparison of the simulated object volume to the calculated volume from FIM measurements taken in the COMSOL model at $0.2 \mathrm{~S} / \mathrm{m}$ bulk conductivity.

\begin{tabular}{|c|c|c|c|c|}
\hline $\begin{array}{c}\text { Object } \\
\text { Conductivity } \\
(\mathrm{S} / \mathrm{m})\end{array}$ & $\begin{array}{c}\text { Simulated volume } \\
\left(\mathrm{m}^{3}\right)\end{array}$ & $\begin{array}{c}\text { Depth } \\
(\mathrm{cm})\end{array}$ & $\begin{array}{c}\text { Calculated } \\
\text { volume }\left(\mathrm{m}^{3}\right)\end{array}$ & Error $(\%)$ \\
\hline 2 & $6.5 \mathrm{E}-05$ & 4.5 & $6.8 \mathrm{E}-05$ & 3.32 \\
\hline 2 & $1.1 \mathrm{E}-04$ & 4 & $1.2 \mathrm{E}-04$ & 2.82 \\
\hline 0.02 & $6.5 \mathrm{E}-05$ & 4.5 & $6.3 \mathrm{E}-05$ & 3.02 \\
\hline 0.02 & $1.1 \mathrm{E}-04$ & 4 & $1.1 \mathrm{E}-04$ & 0.98 \\
\hline 0.4 & $8.7 \mathrm{E}-05$ & 3.5 & $9.0 \mathrm{E}-05$ & 3.61 \\
\hline 0.1 & $1.1 \mathrm{E}-04$ & 5 & $1.1 \mathrm{E}-04$ & 1.83 \\
\hline
\end{tabular}

different depths. The focused impedance values at two electrode separations were obtained and the volume of the object was then calculated using equation (8).

To validate the method experimentally, measurements were performed on a cubic tank filled with saline having dimensions identical to that used in the simulation study. Insulating spherical objects of known volume were placed within the tank and focused impedance values were measured. In figure 6 , $A$ is plotted against inverse of depth which shows almost a linear dependence as in the simulation study. For objects of apparently unknown volume, focused impedance values at two electrode separations were measured and then their volumes were calculated using equation (8). Table 2 summarizes the results obtained from the real life measurements. Here the error was slightly greater, but no more than $7 \%$.

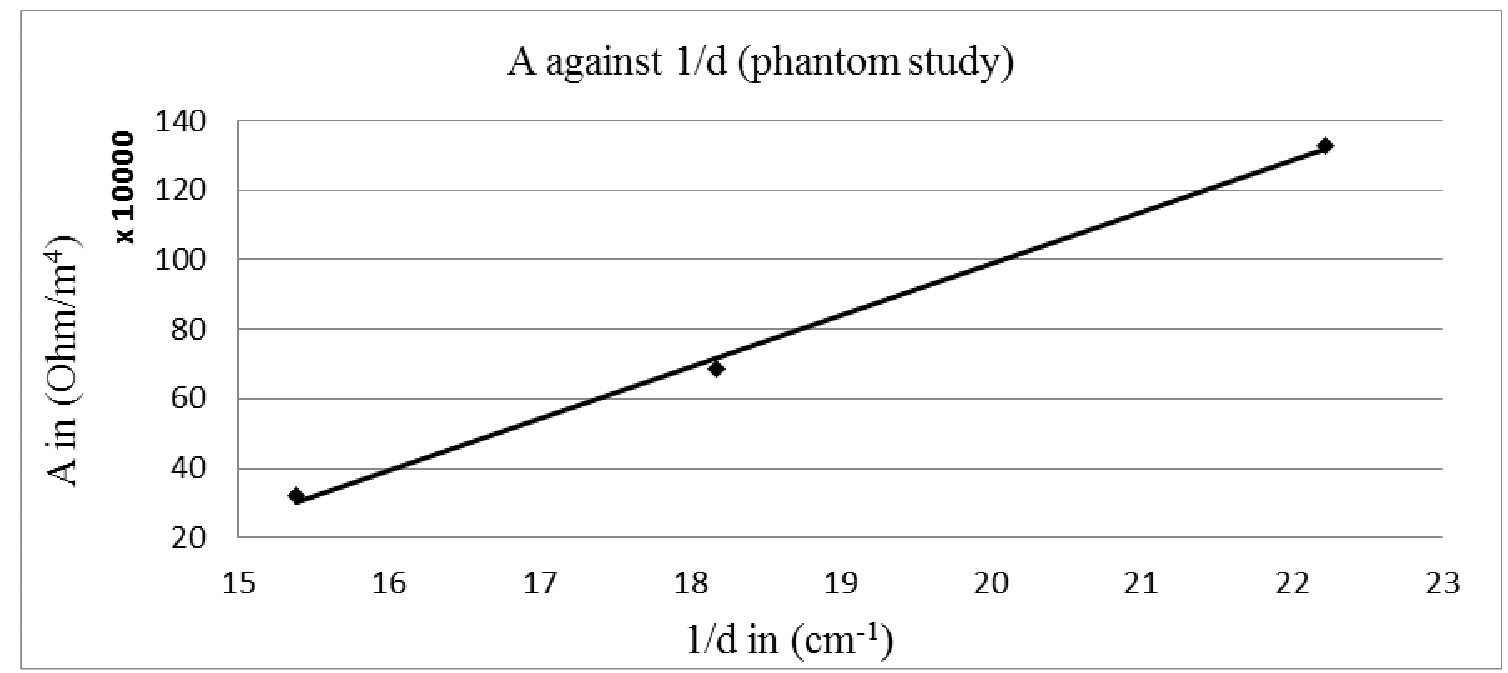

Figure 6: Variation of A against inverse of depth for an insulating spherical object embedded within a cubic tank filled with saline, in a real life experiment 
Table-2: Comparison of the insulating actual object volume to the calculated volume from phantom experiment of FIM measurement taken in the laboratory

\begin{tabular}{|c|c|c|c|}
\hline Actual volume in $\mathrm{m}^{3}$ & Depth in $\mathrm{m}$ & Calculated volume in $\mathrm{m}^{3}$ & \% error \\
\hline $3.1058 \times 10^{-5}$ & 0.045 & $3.0683 \times 10^{-5}$ & 1.21 \\
\hline $1.2478 \times 10^{-4}$ & 0.055 & $1.1662 \times 10^{-4}$ & 6.54 \\
\hline $7.2380 \times 10^{-6}$ & 0.055 & $6.8298 \times 10^{-6}$ & 5.64 \\
\hline
\end{tabular}

\section{DISCUSSIONS AND CONCLUSIONS}

The present work has put forward a new technique for measurement of the volume of an object embedded within a volume conductor using 4-electrode FIM, based on Finite Element simulations. The method has also been verified successfully using both simulated and real life phantom experiments. This technique uses two different electrode separations of FIM and is expected to be useful for objects at reasonable depths from the surface. Of course this technique requires the object depth from the electrode surface to be known, and in case of large organs inside the human body, anatomy can provide this information. This also needs the conductivity values of the target organ with respect to that of the background volume conductor, which has been taken as uniform in the present study. For real life situations in the human body, this latter situation of a background with uniform conductivity will not hold, however, to a certain degree of accuracy, this assumption may give useful results. Again, the conductivity of the target organ should also be available through previous measurements on many subjects.

Although the study was performed only for spherical objects inserted in a homogeneous and isotropic volume conductor, the proposed technique has the potential of giving useful results even when the shape deviates somewhat from a sphere. Thus for estimating volume of an organ within the human body, for instance, estimating the volume of gastric acid in stomach, the volume of urine in bladder, etc., this new method may be useful.

The simulated measurements described in this section were performed over a limited range of object diameters and depths. The ranges used can be approximately expressed as $0<2 \mathrm{r}<\mathrm{ES}_{1}$ and $0<\mathrm{d}<\mathrm{ES} S_{1}$, where $r$ is the radius of the object and $d$ is the depth of the center of the object with respect to the electrode surface, $\mathrm{ES}_{1}$ being the smaller of the two electrode separations of the 4-electrode FIM used. Whether it is applicable to a wider range has to be found out through further work.

The close agreement between the initial assumed radius and the calculated value obtained through the simulated FIM measurements as well as experimental results speaks of success of the new technique of FIM with dual electrode separations. The new technique for volume measurement using FIM will have applications in many areas including Biomedical Physics \& Engineering, geology, oceanography, and industry.

\section{ACKNOWLEDGEMENTS}

The authors gratefully acknowledge the support from the International Science Programme (ISP), Uppsala University, Sweden in procuring a license for COMSOL and for subsistence support to some of the authors. 


\section{REFERENCES}

Al Amin A, Parvin S, Kadir M A, Tahmid T, Alam S K and Rabbani K S 2014 Classification of breast tumour using electrical impedance and machine learning techniques Physiological measurement 35, 965-974

Brown B, Wilson A and Bertemes-Filho P 2000 Bipolar and tetrapolar transfer impedance measurements from volume conductor Electronics Letters 36, 2060-2062

Chen M E, Troncoso P, Johnston D, Tang K and Babaian R J 1999 Prostate cancer detection: relationship to prostate size Urology 53, 764-768

Geraghty E, Boone J, Mcgahan J and Jain K 2004 Normal organ volume assessment from abdominal CT Abdominal imaging 29, 482-490

Grantham J J, Torres V E, Chapman A B, Guay-Woodford L M, Bae K T, King Jr B F, Wetzel L H, Baumgarten D A, Kenney P J and Harris P C 2006 Volume progression in polycystic kidney disease New England Journal of Medicine 354, 2122-2130

Hansen J M, Kampmann J, Madsen S N, Skovsted L, Solgaard S, Grytter C, Grqntvedt T and Rasmussen S N 1979 L-thyroxine treatment of diffuse non-toxic goitre evaluated by ultrasonic determination of thyroid volume Clinical endocrinology 10, 1-6

Hersey S and Sachs G 1995 Gastric acid secretion Physiological Reviews 75, 155-190

Heymsfield S B, Fulenwider T, Nordlinger B, Barlow R, Sones P and Kutner M 1979 Accurate measurement of liver, kidney, and spleen volume and mass by computerized axial tomography Annals of internal medicine 90, 185-187

Holder D S 2010 Electrical impedance tomography: methods, history and applications CRC Press

Howden C and Hunt R 1987 Relationship between gastric secretion and infection Gut 28, 96-107

Islam N, Rabbani K S and Wilson A 2010 The sensitivity of focused electrical impedance measurements Physiological measurement 31, S97-S109, doi:10.1088/0967-3334/31/8/S08

Kadir M A, Ahmed S P, Quaderi G D A, Rahman R and Rabbani K S 2013 Application of Focused Impedance Method (FIM) to Determine the Volume of an Object within a Volume Conductor Proceedings of the 2013 COMSOL Conference, Bangalore, India http://www.comsol.com/paper/download/182751/kadir_paper.pdf

Kadir M A, Baig T N and Rabbani K S 2015 Focused impedance method to detect localized lung ventilation disorders in combination with conventional spirometry Biomedical Engineering: Applications, Basis and Communications 27, 1550029

Kadir M A, Ferdous H, Baig T N and Rabbani K S 2010 Ventilation mapping of chest using Focused Impedance Method (FIM) Journal of Physics: Conference Series 2010012031

Lukaski H C and Bolonchuk W W 1988 Estimation of body fluid volumes using tetrapolar bioelectrical impedance measurements Aviation, space, and environmental medicine 59, 1163 1169

Multiphysics C 2012 Comsol Multiphysics 4.3 user's guide Comsol Multiphysics http://nf.nci.org.au/facilities/software/COMSOL/4.3/doc/pdf/mph/COMSOLMultiphysicsUsersGui de.pdf

Rabbani K and Kabir A 1991 Studies on the effect of the third dimension on a two-dimensional electrical impedance tomography system Clinical Physics and Physiological Measurement 12, 393-402 
Rabbani K and Karal M A S 2008 A new four-electrode Focused Impedance Measurement (FIM) system for physiological study Annals of biomedical engineering 36, 1072-1077

Rabbani K, Sarker M, Akond M and Akter T 1999 Focused impedance measurement (FIM): a new technique with improved zone localization Annals of the New York Academy of Sciences 873, 408-420

Rabbani K S and Kadir M A 2011 Possible Applications of Focussed Impedance Method (FIM) in biomedical and other areas of study Bangladesh Journal of Medical Physics 4, 67-74

Rigaud B, Morucci J P and Chauveau N 1995 Bioelectrical impedance techniques in medicine. Part I: Bioimpedance measurement. Second section: impedance spectrometry Critical reviews in biomedical engineering 24, 257-351

Ryle J A 1926 Gastric Function in Health and Disease Oxford University Press

Shoup M, Gonen M, D'angelica M, Farnagin W R, Dematteo R P, Schwartz L H, Tuorto S, Blumgart L H and Fong Y 2003 Volumetric analysis predicts hepatic dysfunction in patients undergoing major liver resection Journal of gastrointestinal surgery 7, 325-330

Surovy N J, Billah M M, Haowlader S, Al-Quaderi G D and Rabbani K S 2012 Determination of abdominal fat thickness using dual electrode separation in the focused impedance method (FIM) Physiological measurement 33, 707

Tauxe W, Soussaline F, Todd-Pokropek A, Cao A, Collard P, Richard S, Raynaud C and Itti R 1982 Determination of organ volume by single-photon emission tomography. Journal of nuclear medicine: official publication Society of Nuclear Medicine 23, 984-987

Tsuruta M, Nagayama Y, Yokoyama N, Izumi M and Nagataki S 1993 Long-term follow-up studies on iodine-131 treatment of hyperthyroid Graves' disease based on the measurement of thyroid volume by ultrasonography Annals of nuclear medicine 7, 193-197 